\title{
EFFECTS OF IRON DEFICIENCY ANEMIA IN SURGICAL SITE INFECTION
}

\author{
Muhammad Umair Zulfiqar, Umer Fayyaz Ghani, Ayub Ashraf Mallhi*, Khalid Mahmood*, Maria Iqbal**, Muhammad Ans \\ Pak Emirates Military Hospital/National University of Medical Sciences (NUMS) Rawalpindi Pakistan, *Combined Military Hospital/National University of \\ Medical Sciences (NUMS) Rawalpindi Pakistan, ${ }^{* *}$ Bahawal Victoria Hospital, Bahawalpur Pakistan
}

\begin{abstract}
Objective: To determine effect of iron deficiency anemia on the patients developing surgical site infection at surgical department of a tertiary care setup in Pakistan.

Study Design: Comparative prospective study.

Place and Duration of Study: Department of Surgery, Pak Emirates Military Hospital Rawalpindi, from Nov 2018 to Mar 2019. Methodology: A total of 152 indoor hospitalized patients were included in the study. Surgical site infection was assessed by a consultant surgeon according to definitions provided by the Centers for Disease Control and Prevention (CDC) National nosocomial infections surveillance system. Iron deficiency anemia was classed on the basis of hemoglobin and ferritin levels.

Results: Eighty six (56.6\%) patients were male while $66(43.4 \%)$ patients included in the study were female. Commonest surgical procedure was laparotomy 29 (19.1\%) followed by the hernioplasty 27 (17.7\%). Out of 152 patients undergoing surgery and admitted in ward, 35 (23.1\%) showed the presence of surgical site infection while 117 (76.9\%) had no infection. Seventy eight $(51.3 \%)$ patients had iron deficiency anemia while 74 (48.7\%) patients were not anemic. History of transfusion during the surgery and presence of iron deficiency anemia was significantly associated with surgical site infection in the target population.

Conclusion: This study exhibited high frequency of surgical site infection in the target population. Regular screening for anemia should be performed at the surgery department and patients with history of transfusion during the surgery should be looked after especially. Iron deficiency anemia emerged as an independent factor associated with presence of surgical site infection among the surgical patients.
\end{abstract}

Keywords: Iron deficiency anemia, Surgical site infection, Socio-demographic factors.

\footnotetext{
This is an Open Access article distributed under the terms of the Creative Commons Attribution License (https://creativecommons.org/licenses/by-nc/4.0/), which permits
} unrestricted use, distribution, and reproduction in any medium, provided the original work is properly cited.

\section{INTRODUCTION}

One of the major problem especially among females of developing countries is iron deficiency anemia ${ }^{1}$. Many medical, gynecological and neurology associated diseases exert their effect over the blood indices of the individual. Some of the major illnesses associated with iron deficiency anemia are chronic renal disease, liver disease, autoimmune disease, migraine and endocrinopathies among the individuals ${ }^{2-5}$. Infection at surgical site is a fairly common development in most of the surgeries in all parts of the world 6 . Low and middle income countries have been affected more with this untoward phenomenon after the routine surgeries ${ }^{7}$. Multiple factors contribute towards the emergence of this adverse effect in the surgical patients including patient related, illness related, procedure related and post procedure care related factors 8,9 .

Relationship of iron deficiency anemia with the surgical site infection among the surgical inpatients has been reported previously as well in different areas of the world. White et al deduced that anemia is associated with the presence of surgical complications

Correspondence: Dr Muhammad Umair Zulfiqar, Department of Surgery, Pak Emirates Military Hospital, Rawalpindi Pakistan

Received: 30 Jan 2020; revised received: 24 Feb 2020; accepted: 28 Feb 2020 including the surgical site infection in a study done in Africa involving the low resource setting ${ }^{10}$.

Locally present data is not sufficient about surgical site infections among the surgery patients. Studies are available on the epidemiology of anemia in surgical patients of Pakistan, but limited workhave evaluated the presence of iron deficiency anemia among these individuals undergoing various types of surgeries and developing the surgical site infections. Aim of our study was to determine effect of iron deficiency anemia on the patients developing surgical site infection at surgical department of tertiary setup in Pakistan.

\section{METHODOLOGY}

This comparative prospective study conducted in the Surgical department of Pak Emirates Military Hospital (PEMH) Rawalpindi, from November 2018 to March 2019. We calculated sample size by using WHO Calculator with population prevalence proportion of $85 \%{ }^{11}$. We used Non probability consecutive sampling technique for sampling. Inclusion criteria consisted of patients between 18 and 60 years of age who underwent any type of surgery and were admitted in the ward. Patients with any chronic illness other than iron deficiency anemia were excluded. Patients who were undergoing second surgery in less than one month 
time were also excluded. Patients with bleeding disorders, leukemia and lymphomas were also part of the exclusion criteria. Immuno-compromised patients, auto-immune disorder patients and patients on long term steroids were also not included. Pregnant ladies and illicit drug users were excluded at the first step.

Approval was obtained from ethics board committee (ref ltr number: A/28/EC/57/19) and informed written consent was sought from patients who underwent any type of surgery PEMH Rawalpindi surgery department after fulfilling inclusion and exclusion criteria. Infection at surgical site was assessed by surgical consultant keeping in view the definitions provided by the Centers for Disease Control and Prevention (CDC) NNIS system. Relationship of age, gender, education, transfusion during surgery and presence of anemia was determined in association with wound complications among patients undergoing surgeries at PEMH Rawalpindi A special form was designed which included socio demographic profile and all possible infections of wound after surgeries. Education was classed as less than matriculate or matriculate or more.

Iron deficiency anemia was defined as

Blood hemoglobin values of $<12 \mathrm{~g} / \mathrm{dl}$

Serum ferritin level $15 \mathrm{ng} / \mathrm{mL} 1712$

SPSS-24 was used for statistical analysis. Percentages and frequency for gender and anemic patients were calculated. Standard deviation and mean for age was calculated for all the included sample. Relationship of age, gender, education, transfusion during surgery and presence of anemia was analyzed with surgical site infections in patients undergoing surgeries by chi-square test.

\section{RESULTS}

A total of 166 patients were included to take part in our study. Five were not able to consent. Four had some chronic disease and five had co morbid illicit substance use Therefore, total 152 patients contributed in the end. Patients mean age was $40.23 \pm 4.747$ years. $86(56.6 \%)$ patients were males and $66(43.4 \%)$ were females. Out of 152 patients undergoing surgery, 35 $(23.1 \%)$ showed the presence of surgical site infection while $117(76.9 \%)$ had no infection. Seventy eight (51.3\%) patients had iron deficiency anemia while 74 $(48.7 \%)$ patients were not anemic. We applied logistic regression after the application of chi-square and found history of transfusion during the surgery and presence of iron deficiency anemia are interlinked with surgical site infection in patients undergoing surgeries at our tertiary care hospital during the study period (table).

Table: Study participants characteristics $(\mathbf{n}=152)$.

\begin{tabular}{l|c}
\hline \multicolumn{2}{l}{ Age (years) } \\
\hline Mean \pm SD & $40.23 \pm 4.747$ \\
Range (Min-Max) & $19-60$ Years \\
\hline Gender & $86(56.6 \%)$ \\
\hline Male & $66(43.4 \%)$ \\
Female & $29(19.1 \%)$ \\
\hline Surgeries Performed & $27(17.7 \%)$ \\
Laparotomy & $24(15.8 \%)$ \\
Hernioplasty & $22(14.4 \%)$ \\
Open Cholecystectomy & $22(14.4 \%)$ \\
Laparoscopic Cholecystectomy & $19(12.5 \%)$ \\
Appendectomy & $09(5.9 \%)$ \\
hemorrhoidectomy &
\end{tabular}

\section{DISCUSSION}

This study was novel because it incorporated one surgical condition and one common medical condition and look for the factors affecting them. Association between anemia and surgical site infections has been established in different studies previously in different parts of the world by lieu et al and Shaw et al11,13 Using routine laboratory technique, we estimated that $>50 \%$ of our surgical patients exhibited presence of iron deficiency anemia including patients of both genders and of adult age group. Some factors that can contribute to presence of iron deficiency in these patients can be because of underlying illness requiring surgery, long standing illness or psychological problems leading to nutrition problems and nutritional deficiencies in general ${ }^{11-13,14}$. Reason behind this can be linked to physiology of anemia and underlying surgical condition, disease chronicity and some medications side effects. Sample population in our study consisted of patients from a developing country which have a lot of confounding variable predisposing them to anemia. Repeated assessments and longitudinal studies are needed to differentiate iron deficiency anemia due to underlying surgical pathology or other factors.

Another study done on the patients undergoing elective colorectal surgery reproduced similar findings ${ }^{11}$. A recent meta-analysis concluded that anemia is an independent risk factor predicting the increased overall mortality after the surgery. Infection after surgery was also significantly linked with the presence of anemia in surgical patient in different studies included in the meta-analysis ${ }^{12}$.

The process through which infection and anemia are connected is intricate and multifaceted. Iron defi- 
ciency can lead to ineffective immune system and lowers the threshold of individual towards various infections. Similarly there are various infections caused by variety of pathogens which can lead to iron deficiency in the body making the patient anemic ${ }^{13,14}$.

Surgical site infections affect the overall outcome of the surgery inversely and increase the overall mortality of patient after the procedure. It also poses a burden by causing prolong use of antibiotics. Longer hospital stay is also linked with the surgical site infections causing a burden on the individual as well as health care budget of the country ${ }^{15,16}$.

Iron deficiency anemia is a multi-faceted problem with nutritional, physiological and physical parameters. Underlying illness or its medical treatment may alter these parameters in one way or the other. It is interesting fact that sometimes surgical procedure may be required for the cure of anemia and its presence before the surgery can increase the chances of complications as well as reported in our study. This requires a detailed assessment before the surgery and multidisciplinary approach in the management of patient. Normal blood counts and iron indices are essential for quality life ${ }^{17-20}$. Therefore early screening and treatment can lead to improvement in quality of life of a patient who is already suffering from a chronic illness and undergoing surgery.

Various studies in varied parts of the world have produced similar results as that of our study. Iron deficiency anemia prior to the surgical procedure has been correlated with surgical site infection in the patients in these studies by white et al in 2017 and other studies as well ${ }^{10-12}$. Though screening of hemoglobin is a routine investigation before the surgical procedures either elective or emergency and in severe cases anesthetists don't declare the patient fit for surgery especially in the elective cases. Our results emphasize similar phenomenon i.e. if possible elective surgeries should be delayed till blood indices are within normal range to reduce the risk of surgical site infection.

This study consisted of male patients in majority. Previously epidemiological studies conducted at Pakistan by Malik et al and Ali et al have shown male predominance among the surgical site infection patients 17,18 . Relationship of gender though was insignificant with the presence of surgical site infections in our sample. Predominant male sample may be due to the sampling bias as our sample was derived from a tertiary care hospital where most of the entitled patients are the male soldiers and officers. Further studies car- ried out in public sector hospital may throw more light on this phenomenon. History of blood transfusion was associated with the increased risk of surgical site infections among our study patients undergoing surgery at our department. Similar results were reported by studies done in other settings by $\mathrm{Hu}$ et al and Strauss et al in 201819,20. Reason might be transfusion related immunosuppression via interleukin pathway or longer duration of surgery due to blood transfusion.

One of the limitation of our study was not being able to select patients randomly. Therefore, our study results may not be generalized on general population. Since our study was cross-sectional, Therefore the cause and effect relationships is not clear. Future studies are therefore warranted to establish associations with the help of longitudinal epidemiological data. Our study did not include control and severity of all the co morbid medical illnesses as many diseases may not directly but indirectly can lead to anemia as well as surgical site infection. Moreover socio-economic and nutritional status may serve as confounding factor which was not addressed. Therefore longitudinal studies with sophisticated study design and bigger sample size are proposed to confirm association of surgical site infection, iron deficiency anemia and associated risk factors.

\section{CONCLUSION}

Iron deficiency anemia was quite prevalent among patients who suffer from infection at surgical sites. Routine screening of anemia should be done biannually and patients with chronic illnesses and nutritional deficiencies should be screened at earliest visits.

\section{CONFLICT OF INTEREST}

This study has no conflict of interest to be declared by any author.

\section{REFERENCES}

1. Soofi S, Khan GN, Sadiq K. Prevalence and possible factors associated with anaemia, and vitamin B12 and folate deficiencies in women of reproductive age in Pakistan: analysis of nationallevel secondary survey data. BMJ Open 2017; 7(12): e018007.

2. Keivani Z, Mirzaei M, Mahmoudzadeh M. The relationship between migraine headache and iron deficiency anemia in patients referred to neurology clinic of shahrekord university of medical sciences. Iran J Nurs 2010; 23(64): 37-43.

3. Johnson-Wimbley TD, Graham DY. Diagnosis and management of iron deficiency anemia in the $21^{\text {st }}$ century. Therap Adv Gastroenterol 2011; 4(3): 177-84.

4. Gkamprela E, Deutsch M, Pectasides D. Iron deficiency anemia in chronic liver disease: etiopathogenesis, diagnosis and treatment. Ann Gastroenterol 2017; 30(4): 405-13.

5. Aoun M, Karam R, Sleilaty G, Antoun L, Ammar W. Iron deficiency across chronic kidney disease stages: Is there a reverse gender pattern? Barretti P, ed. PLoS ONE 2018; 13(1): e0191541. 
6. Baker AW, Dicks KV, Durkin MJ, Weber DJ, Lewis SS. Epidemiology of surgical site infection in a community hospital network. Infect Control Hosp Epidemiol 2016; 37(5): 519-26.

7. Pathak A, Mahadik K, Swami MB, Roy PK, Sharma M, Mahadik $\mathrm{VK}$, et al. Incidence and risk factors for surgical site infections in obstetric and gynecological surgeries from a teaching hospital in rural India. Antimicrob Resist Infect Control 2017; 6(2): 66.

8. Carvalho RLR, Campos CC, Franco LMC, Rocha AM, Ercole FF. Incidence and risk factors for surgical site infection in general surgeries. Rev Lat Am Enfermagem 2017; 25(3): e2848.

9. Rajkumari N, Sharma K, Mathu P, Kumar S, Gupta A. A study on surgical site infections after trauma surgeries in a tertiary care hospital in north India. Indian J Med Res 2014; 140(5): 691-94.

10. White MC, Longstaff L, Lai PS. Effect of pre-operative anaemia on post-operative complications in low-resource settings. World J Surg 2017; 41(3): 644-49.

11. Liu L, Liu L, Liang LC, Zhu ZQ, Wan X, Dai HB, et al. Impact of preoperative anemia on perioperative outcomes in patients undergoing elective colorectal surgery. Gastroenterol Res Pract 2018; 2018: 2417028.

12. Fowler AJ, Ahmad T, Phull MK, Allard S, Gillies MA, Pearse RM. Meta-analysis of the association between preoperative anaemia and mortality after surgery. Br J Surg 2015; 102(11): 1314-24.

13. Shaw JG. Iron deficiency anemia: focus on infec-tious diseases in lesser developed countries. Anemia 2011; 2011 (1): 260380.
14. Viana MB. Anemia and infection: a complex relationship. Rev Bras Hematol Hemoter 2011; 33(2): 90-92.

15. Abdullah HR, Sim YE, Hao Y, Lin GY, Liew GHC, Lamoureux EL, et al. Association between preoperative anaemia with length of hospital stay among patients undergoing primary total knee arthroplasty in Singapore: a single-centre retrospective study. BMJ Open 2017; 7(6): e016403.

16. Olowo-Okere A, Ibrahim YKE, Sani AS, Olayinka BO. Occurrence of surgical site infections at a tertiary healthcare facility in abuja, nigeria: a prospective observational study. Med Sci (Basel) 2018; 6(3): 60.

17. Malik ZI, Nawaz T, Abdullah MT, Waqar SH, Zahid MA. Surgical site infections in general surgical wards at a tertiary care hospital. Pak J Med Res 2013; 52(4): 116-19.

18. Ali SA, Soomro AG, Memon AI, Siddiqui AJ. Prevalence, evaluation and management of preoperative anaemia in the elective general surgical patients. J Ayub Med Coll Abbottabad 2012; 24 (3-4): 59-61.

19. Hu T, Wu X, Hu J, Chen Y, Liu H, Zhou C, et al. Incidence and risk factors for incisional surgical site infection in patients with Crohn's disease undergoing bowel resection. Gastroenterol Rep (Oxf) 2018; 6(3): 189-94.

20. Strauss WE, Auerbach M. Health-related quality of life in patients with iron deficiency anemia: impact of treatment with intravenous iron. Patient Relat Outcome Meas 2018; 9(4): 285-98. 